
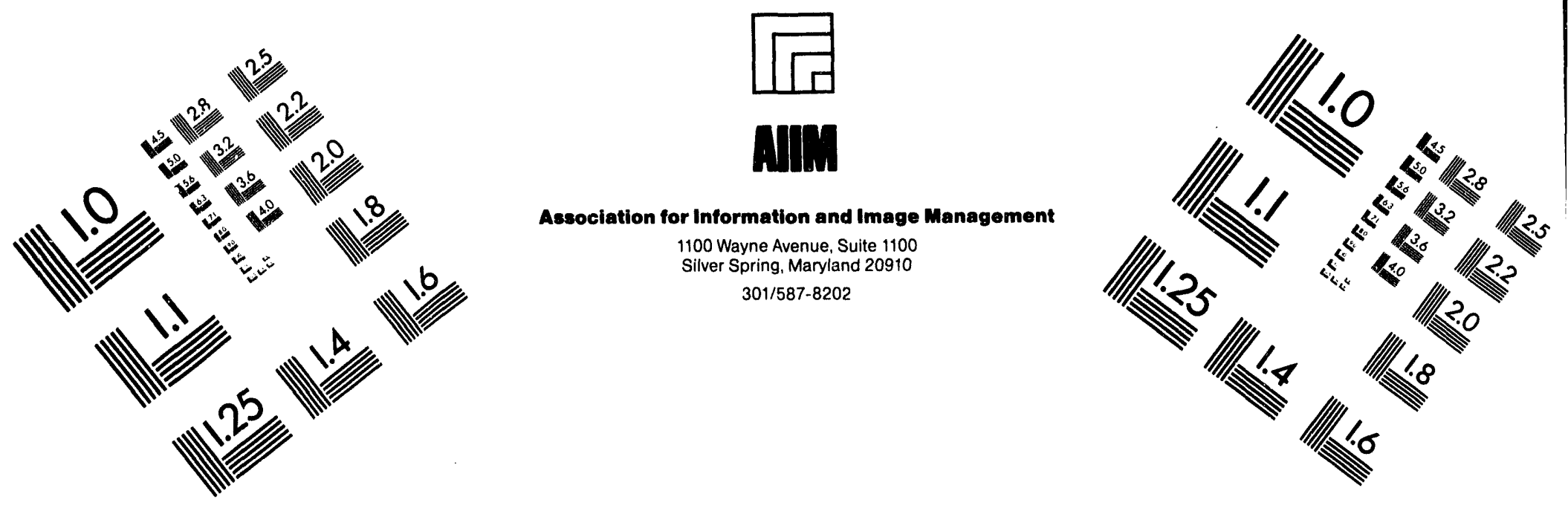

\title{
Centimeter
}

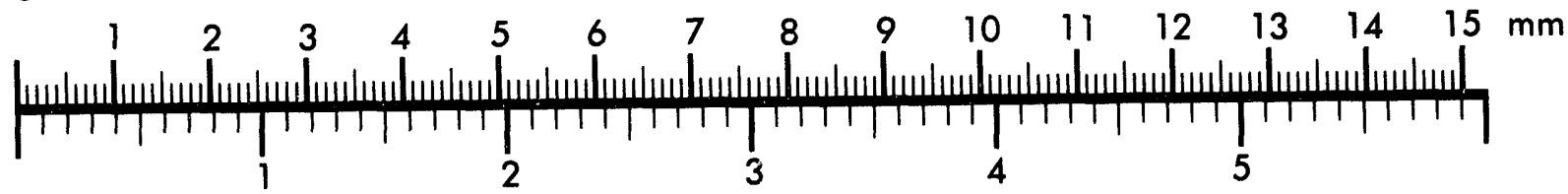

Inches

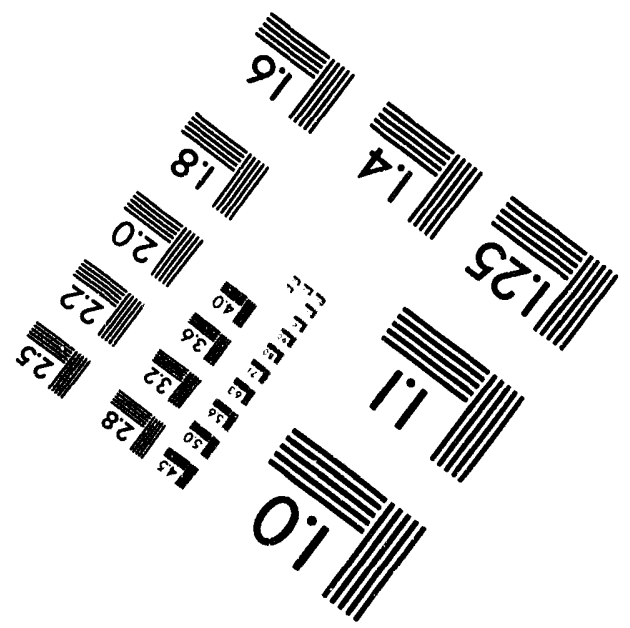

MANUFACTURED TO AIIM STANDARDS

BY APPLIED IMAGE. INC.

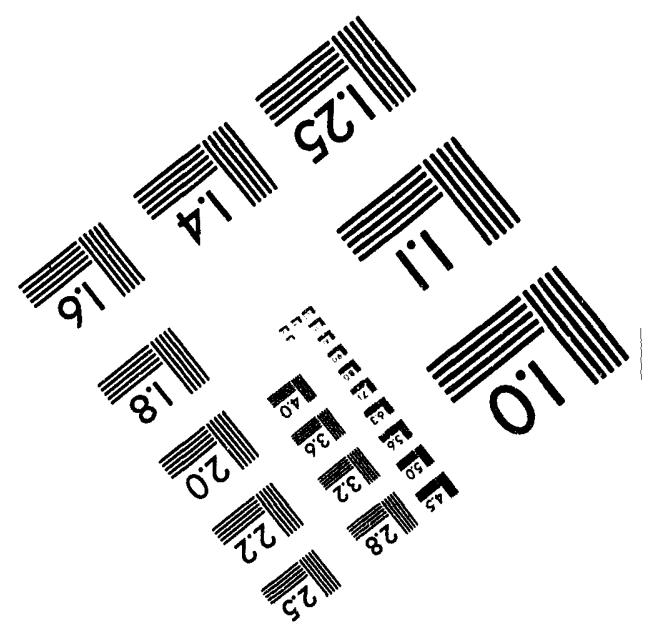



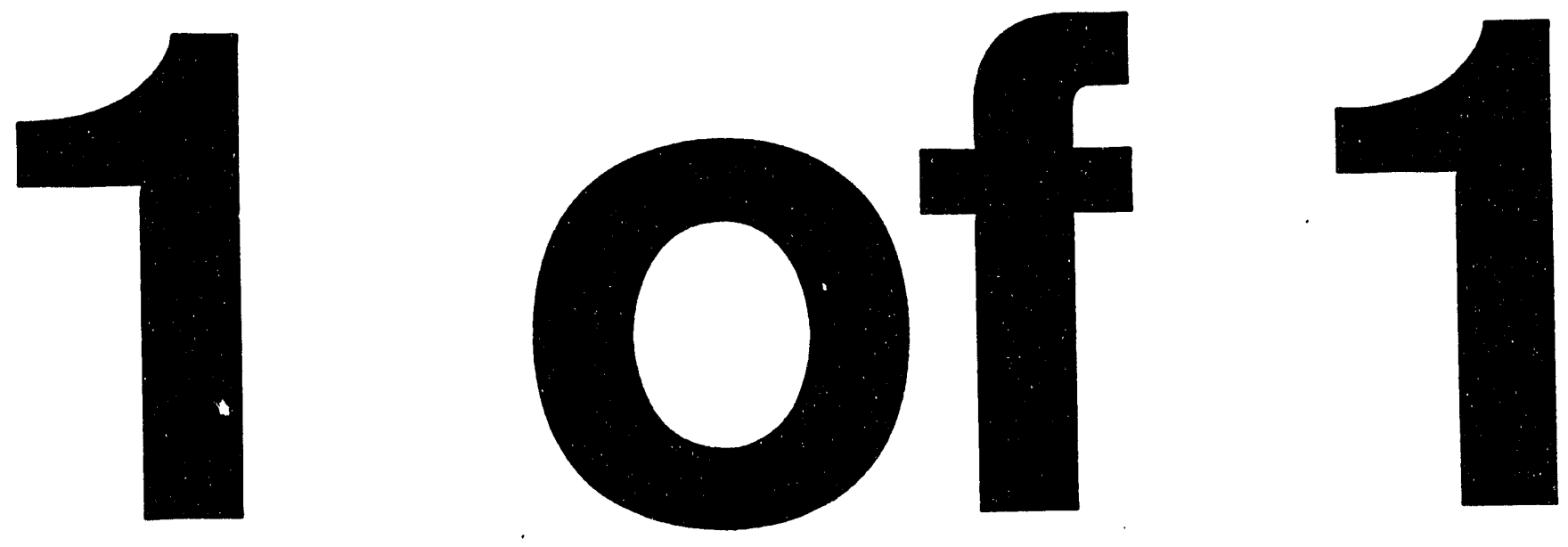


\title{
ANK/TD/CP--82060
$\operatorname{CONF}-940664-23$
}

\section{An Assessment of Disruption Erosion in the ITER Environment*}

\author{
A. Hassanein \\ Argonne National Laboratory \\ 9700 South Cass Avenue \\ *.Argonne, Illinois 60439 USA
}

and

I. Konkashbaev

Troitsk Institute for Innovation

Troitsk, Moscow Region, 142092 Russia

\section{DISCLAIMER}

\begin{abstract}
This report was prepared as an account of work sponsored by an agency of the United States Government. Neither the United States Government nor any agency thereof, nor any of their bility for the accuracy warranty, express or implied, or assumes any legal liability or responsiprocess disced accuracy, completeness, or usefulness of any information, apparatus, product, or process disclosed, or represents that its use would not infringe privately owned rights. Refermanufacturer, any specific commercial product, process, or service by trade name, trademark, manufacturer, or otherwise does not necessarily constitute or imply its endorsement, recommendation, or favoring by the United States Government or any agency thereof. The views and opinions of authors expressed herein do not necessarily state or reflect those of the United States Government or any agency thereof.
\end{abstract}

August 1994

"Work supported by the United States Department of Energy, Office of Fusion Energy, under Contract W-31-109-Eng-38.

Presented at the Third International Symposium on Fusion Nuclear Technology (ISFNT-3), June 27 - July 1, 1994, Los Angeles, California. 


\title{
An Assessment of Disruption Erosion in the ITER Environment*
}

\author{
A. Hassanein ${ }^{1}$ and I. Konkashbaev ${ }^{2}$
}

${ }^{1}$ Argonne National Laboratory, 9700 South Cass Ave., Argonne, IL USA 2Troitsk Institute for Innovation, Troitsk, Moscow Region, 142092 Russia

\section{ABSTRACT}

The behavior of divertor materials during a major disruption in ITER is very important to successful and reliable operation of the reactor. Erosion of material surfaces due to a thermal energy dump can severely limit the lifetimes of plasma-facing components and thus diminish the reactor's economic feasibility. A comprehensive numerical model has been developed and used in this analysis, which includes all major physical processes taking place during plasma/material interactions. Models to account for material thermal evolution, plasma/vapor interaction physics, and models for hydrodynamic radiation transport in the developed vapor cloud are implemented in a self-consistent manner to realistically assess disruption damage. The extent of self-protection from the developed vapor cloud in front of the incoming plasma particles is critically important in determining the r verall disruption lifetime. Models to study detailed effects of the strong magnetic field on the behavior of the vapor cloud and on the net erosion rate have been developed and analyzed. Candidate materials such as beryllium and carbon are both considered in this analysis. The dependence of divertor disruption lifetime on disruption physics and reactor conditions was analyzed and discussed.

*Work supported by the U.S. Department of Energy, Office of Fusion Energy, under Contract No. W-31-109-Eng-38. 


\section{INTRODUCTION}

Disruption damage to plasma-facing materials (PFMs) in a magnetic fusion reactor is a major concern for sate, successful, and reliable reactor operation. The intense deposition of energy $\left(10-200 \mathrm{MJ} / \mathrm{m}^{2}\right)$ over short periods $(0.1-3 \mathrm{~ms})$ will cause severe surface erosion and melting of these components. The exact amount of eroded material is critically important to reactor design and component lifetime analysis. In current tokamak machines, ITER-like heat loads and disruption conditions are not achievable. It is therefore necessary to develop a comprehensive model that includes all major physical processes occurring during a disruption in order to correctly simulate plasma/material interaction in reactor environments and conditions. Modeling of the detailed interaction of plasma particles with the initial ablated material is quite important because the ablated material provides a much-needed shielding layer that protects the PFM from the incident plasma particles.

A recently developed comprehensive model [1] has been enhanced and used in this analysis. In this model, three major modeling stages of plasma/material interaction were developed with sufficient detail to accurately simulate a disruption effect on PFM. Initially, the incident plasma particles from the disrupted plasma will deposit part of their energy on the PFM surface. Models for particle deposition and material thermal evolution that take into account phase change, moving boundaries, and temperature-dependent thermophysical properties, etc., were developed to predict the behavior of these components. This initial burst of energy delivered to PFM surfaces from the direct impact of plasma particles will 
cause sudden ablation of these materials. As a result, a vapor cloud will be formed in front of the incoming plasma particles. Shortly thereafter, the plasma particles will be completely stopped in this vapor cloud. Continuous heating of the vapor cloud will ionize, excite, and generate photon radiation. The initial plasma particle kinetic energy is therefore transformed into radiation energy. Comprehensive models for the hydrodynamics and heating of the vapor cloud that shields the original surface were developed for the second stage of disruption modeling. Finally, models for radiation transport throughout the vapor cloud were developed to estimate the het heat flux transmitted to the facing material. It is therefore the dynamics and evolution of this vapor cloud that will finally determine the net erosion rate at the end of a disruption. Figure 1 is a schematic illustration of the various interaction zones and processes during the plasma/material interaction that follows a disruption under the influence of a strong magnetic field. This problem requires the solution of three moving boundaries: the vapor front, the receding target surface, and the solid/liquid interface. These three moving boundaries are interdependent, and a complete solution should link them dynamically and simultaneously.

In this study, detailed effects of the strong reactor-environment magnetic field on the magnetohydrodynamics of the vapor cloud and on the resulting erosion rate were examined. A two-dimensional (2-D) magnetohydrodynamic model was developed to include effects such as magnetic field diffusion, friction forces, and Joule heating of the vapor material. Previous analysis of the effect of a strong magnetic field on the dynamics of plasma/material interaction were mainly qualitative, used 
simple assumptions, and were not integrated dynamically with other physical processes [2-4]. Candidate PFMs such as beryllium and carbon were considered in this analysis. Disruption lifetime of these materials have been calculated, and dependence on characteristics of the plasma/vapor interaction zone and disruption parameters were analyzed.

\section{MODELING SUMMARY}

\subsection{Plasma/Material Interaction}

The thermal evolution of the PFM is calculated by solving a timedependent heat conduction equation in one- or multidimensional coordinates [5]. All thermophysical properties are assumed to be temperature-dependent. Surface temperature was determined by both the boundary conditions and the dynamics of the evaporation process. The volumetric energy deposition by the incident plasma particles is calculated with detailed models that include slowing-down physics of both plasma ions and piasma electrons [6]. Phase transformation of metallic PFM is taken into account by using detailed models [7]. Kinetic energy of the incident plasma particles, the photon energy radiated by the vapor cloud, vapor-conducted energy, and free-streaming energy (from near-surface vapor to target material) [1] are partitioned inside the PFM into conduction, melting, and evaporation energy. 


\subsection{Plasma/Napor Interaction}

As direct heating of the PFM continues by the impinging plasma particles, the surface temperature rises to the point at which significant ablation begins. The produced vapor leaving the surface will accumulate, expand, and interact with incoming plasma particles. The continuous deposition of energy in the vapor layer by plasma particles will produce intense bulk vapor heating and vapor ionization. The ionized vapor will interact with the strong magnetic field, which then limits expansion of the vapor to mainly along field lines. Additional heating of the original exposed PFM surface is only from vapor thermal radiation, vapor thermal conduction, free-energy streaming, and other enhanced plasma radiation losses [1].

Vapor expansion into the vacuum vessel under the influence of a strong magnetic field is determined by solving the vapor magnetohydrodynamic equations for conservation of mass, momentum, and energy:

$$
\begin{aligned}
& \frac{\partial \rho}{\partial t}+\nabla \cdot(\rho V)=0, \\
& \rho \frac{\partial V}{\partial t}+\nabla P=0,
\end{aligned}
$$

and

$$
\frac{\partial E}{\partial t}+\nabla \cdot(E V)+P \nabla \cdot V=\nabla \cdot(K \nabla T)+\nabla \cdot Q_{r}+\nabla \cdot Q_{b}
$$


where $V$ is vapor velocity, $P$ is density, $E$ is energy, $P$ is pressure, $K$ is vapor conductivity, $Q_{r}$ is radiation flux, and $Q_{b}$ is the incident particle flux from the disrupting plasma. All variables of these equations are both timeand space-dependent. The vapor plasma, once ionized, is assumed to move freely along magnetic field lines ( $r$-direction), as shown in Fig. 1. One needs to solve the vapor equation of motion in two directions; along and perpendicular to divertor surface. The radiation transport equations are not directly affected by the magnetic field and can be solved in one or two dimensions.

$$
+\infty
$$

The vapor equation of motion in a strong magnetic field environment can be written as

$$
\rho \frac{d^{2} r}{d t^{2}}=-\nabla P+J \times B
$$

where $r$ is distance along field lines, $J$ is vapor current density, and $B$ is magnetic flux density. The induced magnetic force $\mathrm{J} \times \mathrm{B}$ acts as a retarding force to vapor expansion. This force mainly acts in the perpendicular direction to magnetic field lines. The magnetic force is assumed to be composed of a magnetic pressure force, $F_{m}$, and a friction force, $F_{c}$, due to the curvature of the magnetic field lines, where

$$
F_{m}=\frac{1}{\mu_{0}} \nabla B^{2}
$$

and 


$$
F_{c}=\frac{1}{\mu_{o}} \frac{B^{2}}{R_{c}}
$$

where $\mu_{0}$ is magnetic permeability and $R_{c}$ is the radius of curvature of magnetic field lines. The current density, $\mathrm{J}$, is given by

$$
J=\frac{1}{\mu_{0}} \nabla \times B
$$

The solution of the magnetohydrodynamic vapor equation of motion takes into account magnetic field diffusion in the vapor cloud. The variation of the magnetic field with time can be written as

$$
\frac{\partial B}{\partial t}=-\frac{1}{\mu_{0}} \nabla \times\{E+V \times B\}
$$

and

$$
E=\frac{J}{\sigma}
$$

where $\sigma$ is vapor conductivity. For a weakly ionized low-temperature, high-density vapor-plasma, the conductivity is given by

$$
\sigma=\frac{4 \pi n_{e} e^{2}}{m_{e}} \tau
$$

where $n_{e}$ is density, $e$ is charge, $m_{e}$ is mass of the electron, and $\tau$ is the vapor plasma collision time. The above equations are solved in 2-D coordinates, i.e., along and across the magnetic field lines. The solution is 
then transformed to the $x$ (along divertor surface) - $y$ (normal to divertor surface) coordinates, as shown in Fig. 1. Joule heating of the vapor cloud, which is given by $\mathrm{J}^{2} / \sigma$, is also taken into account in these calculations.

\subsection{Radiation Transport}

After enough vapor accumulates in front of the incoming plasma particles, the plasma particles are then stopped in the vapor cloud, thereby heating and ionizing the cloud. Continuous vapor heating by the plasma particles will cause the vapor to emit photon radiation. The plasma energy is then transmitted indirectly to PFM surfaces through photon radiation. Therefore, radiation transport plays an important role in determining the final erosion rate of the PFM and consequently the component lifetime. The radiation transport equations are solved by the forward-reverse method [8]. The radiation is composed of two separate fluxes, continuum radiation and line radiation. The most intense lines are treated separately, while the less intense lines are combined with the continuum radiation flux $[1,9]$. Opacity and emissivity data are provided in the form of look-up tables for a wide range of expected vapor densities and temperatures.

\section{ANALYSIS}

The models described above, including that for the effect of a strong magnetic field on vapor cloud hydrodynamics, are implemented in a new optimized version of the computer code A*THERMAL-S [1]. Thermal quench time during the disruption is assumed in this analysis to be $100 \mu \mathrm{s}$. Calculations for radiation transport and vapor magnetohydrodynamics are 
extended up to $10 \mu$ s beyond the disruptic n time to realistically simulate a situation where the vapor and the radiation flux cannot immediately disappear just after the disruption. D'sruption energy densities of $10-100 \mathrm{MJ} / \mathrm{m}^{2}$ are used in this analysis.

Figure 2 shows the effect of a 5-T magnetic field on carbon erosion rate during a $100-\mu$ s disruption with an energy density of $10 \mathrm{MJ} / \mathrm{m}^{2}$. The inclined magnetic field reduced the erosion rate in this case by about $30 \%$. The magnetic field keeps the vapor cloud more dense and closer to the surface, thus more radiation is absorbed in the vapor and less radiation is transported to the PFM surface. A similar effect is predicted for a disruption on beryllium, as shown in Fig. 3. The magnetic field reduces both erosion rate and melt layer thickness by a factor of 2 . The resulting melt layer thickness is much greater than the ablation thickness, which causes a serious concern if the melt layer is lost during the disruption due to the various forces on the melt layer during the disruption [10]. The magnetic field is found to be more effective in reducing disruption erosion of beryllium than that of carbon. In fact, where disruption energy density is high, the magnetic field can slightly increase the carbon erosion rate. This is mainly because carbon is a better radiative material than beryllium.

Figure 4 shows the vapor front temperature of both beryllium and carbon vapor during a disruption. Carbon, being a higher-Z material, radiates more el,ergy than beryllium, which results in lower vapor temperature at these disruption parameters. At higher disruption power densities, however, carbon vapor front temperature can exceed that of beryllium due to different radiation physics phenomena. The higher 
radiation rate of carbon (radiation cooling) causes more radiation flux to be emitted toward the PFM, which in turn causes more material to be ablated. For the same disruption conditions given in this case, the carbon ablation rate is about two to three times that of beryllium. Vapor shielding is generally expected to be more effective in reducing net energy flux to lower-Z PFM materials than to higher-Z materials.

Figure 5 shows beryllium vapor density and temperature in relationship to normal distance above the divertor plate with and without a magnetic field. In the absence of a magnetic field, the ablated material expands freely - opposed only by plasma particle momentum - in the normal direction. Once the vapor is ionized in a magnetic field environment its motion follows field lines. Because of the oblique angle of the field lines, vapor expansion normal to the surface is substantially reduced. As a result, vapor density near the divertor plate is much higher which tends to provide better shielding to the PFM surface. However, this higher vapor density tends to increase processes such as vapor conduction, turbulence, and instabilities that can substantially increase the erosion rate. Figure 6 shows the expansion velocity components in the $x$ and $y$ directions under the influence of a 5-T magnetic field. Vapor expansion along the divertor surface is much higher ( $\approx 40$ times) than in the normal direction. Initially, the normal velocity is higher because the ablated material leaves the surface as neutrals in the normal direction and is not affected by the magnetic field until it is ionized.

Figure 7 compares beryllium and carbon vapor density and temperature as a function of normal distance above the divertor plate for 
the same disruption conditions. Carbon vapor has a higher density and lower temperature, and expands farther in the normal direction, than beryllium. The higher normal expansion is due mainly to the more powerful pressure gradient of the ablated carbon. The lower temperature is mainily due to the increased radiation emitted from the higher- $Z$ carbon vapor. Because of this lower temperature, carbon vapor expansion along the divertor surface is lower than that for beryllium. This is clearly shown in Fig. 8, where the expansion velocity along the divertor surface $\left(V_{x}\right)$ is about $50 \%$ of that predicted for beryllium (Fig. 6).

The incident plasma energy density on the ITER divertor plate during a disruption can be $\geq 100 \mathrm{MJ} / \mathrm{m}^{2}$. This can occur if the disrupted plasma deposits its energy only on parts of the toroidal divertor plate. Because of the high heat load expected during normal operations, and due to design limitations on the maximum allowable surface temperature, it is assumed in this study that typical initial thicknesses of beryllium coating and carbon tiles are 3 and $10 \mathrm{~mm}$, respectively. It is further assumed that $50 \%$ of this initial thickness can be sacrificed to disruption erosion before repair of the beryllium coating or replacement of the carbon tiles becomes necessary. Figure 9 shows the maximum allowable number of disruptions for beryllium and carbon materials at various incident plasma energy densities. If the beryllium melt layer is lost during the disruption, the beryllium disruption lifetime is only about 50 disruptions. If the melt layer is lost as soon as it develops, however, disruption lifetime can be severely shortened. A one-order-of-magnitude increase in energy density reduces the disruption ablation lifetime by a factor of only $\approx 2$. This is because higher incident plasma energy densities are mainly used to heat the front 
regions of the vapor and only a small fraction of this energy is transmitted to and deposited on the PFM surface [1].

The effect of plasma particle kinetic energy on erosion thickness is less important ir a magnetic field environment. This is mainly because of the oblique angle of incidence of the magnetic field lines $\left(\approx 2^{\circ}-5^{\circ}\right)$ to the divertor plate surface. This substantially shortens the range of the plasma particles in both the PFM and the ablated material, resulting in more surface energy deposition rather than volumetric deposition [1]. However, if a sheath potential is developed during disruption, plasma particles can be accelerated to much higher energies. The magnetic field helps reduce the 2-D radiation transport losses because it confines the vapor cloud near the surface of the divertor plate. However, this tends to increase the erosion rate because less radiation escapes to the vacuum chamber away from the PFM surface [9].

Additional analysis is required for several important issues that can affect net erosion rate and consequently the lifetime of the divertor plate. Uncertainties in disruption parameters such as incident energy density and disruption time are very important in determining ablation rate, melt-layer thickness, development of melt-layer instabilities, and potential loss of the melt layer [10]. Vapor thermal conduction with turbulence, as well als development of vapor instabilities, may significantly increase heat flux to the PFM, resulting in a large increase in erosion rate. Magnetic field edge effects should be investigated where the ablated material is not fully ionized and can escape confinement by the magnetic field, resulting in r.uch less shielding for the PFM. 


\section{CONCLUSIONS}

A two-dimensional magnetohydrodynamic model is developed to take into account detailed interaction physics of the vapor cloud with the reactor magnetic field. Included are effects such as magnetic field diffusion, induced electric field, vapor conductivity, and Joule heating of the vapor. This model is integrated with a recently developed cumprehensive model that realistically solves the problem of three moving boundaries, i.e., vapor expansion, surface recession, and liquid metal propagation front. Models for material thermal evolution with phase change, vapor magnetohydrodynamics, and radiation transport are dynamically linked, integrated, and optimized for realistic evaluation of disruption effects in reactor environment.

The reactor magnetic field may help reduce erosion rates during a disruption at lower energy densities. The inclined field, however, will reduce 2-D radiation transport losses which have the effect of increasing erosion rates particularly for higher energy densities. Edge effects due to oblique incidence of the magnetic field may reduce vapor accumulation in front of the incoming plasma, which has the effect of increasing erosion rates. In addition, higher vapor density due to magnetic field confinement enhances processes such as vapor conduction, turbulence, and instabilities which all tend to increase erosion rates. 


\section{REFERENCES}

1. A. Hassanein and I. Konkashbaev, "Comprehensive Model for Disruption Erosion in a Reactor Environment," presented at the 11th International Conference on Plasma-Surface Interactions, Mito, Japan, May 22-27, 1994. To be published in J. Nucl. Mater.

2. A. Sestero and A. Ventura, J. Nucl. Mater. 128 \& 129 (1984) 828.

3. J. Gilligan and D. Hahn, J. Nucl. Mater. 145-147 (1987) 391.

4. H. Bolt et al., J. Nucl. Mater. 196-198 (1992) 948.

5. A. Hassanein, J. Nucl. Mater. 122 \& 123 (1984) 1453.

6. A. Hassanein and D. Ehst, J. Nucl. Mater. 196-198 (1992) 680.

7. A. Hassanein, ASME, 88-WA/NE-2.

8. B.N. Chetverushkin, Mathematical modelling of the radiative gas, Nauka, Moscow (1986).

9. A. Hassanein, "Plasma Disruption Modeling and Simulation," an invited paper presented at the 11th Topical Meeting on the Technology of Fusion Energy, New Orleans, LA, June 19-23, 1994.

10. A. Hassanein, Fusion Technology 15 (1989) 513. 


\section{FIGURE CAPTIONS}

1. Schematic illustration of various interaction processes in a strong magnetic field during a disruption.

2. Carbon erosion rate with and without magnetic field effect during a disruption.

3. Beryllium erosion rate and melting thickness with and without magnetic field effect dưfing a disruption.

4. Front vapor-cloud temperature for both beryllium and carbon during a disruption.

5. Effect of magnetic field on beryllium vapor temperature and density.

6. Beryllium vapor expansion velocities along $\left(V_{x}\right)$ and normal $\left(V_{y}\right)$ to divertor surface.

7. Beryllium and carbon vapor density and temperature as a function of distance normal to surface.

8. Carbon vapor expansion velocities along $\left(V_{x}\right)$ and normal $\left(V_{y}\right)$ to divertor surface.

9. Maximum allowable number of disruptions for various incident energy densities. 


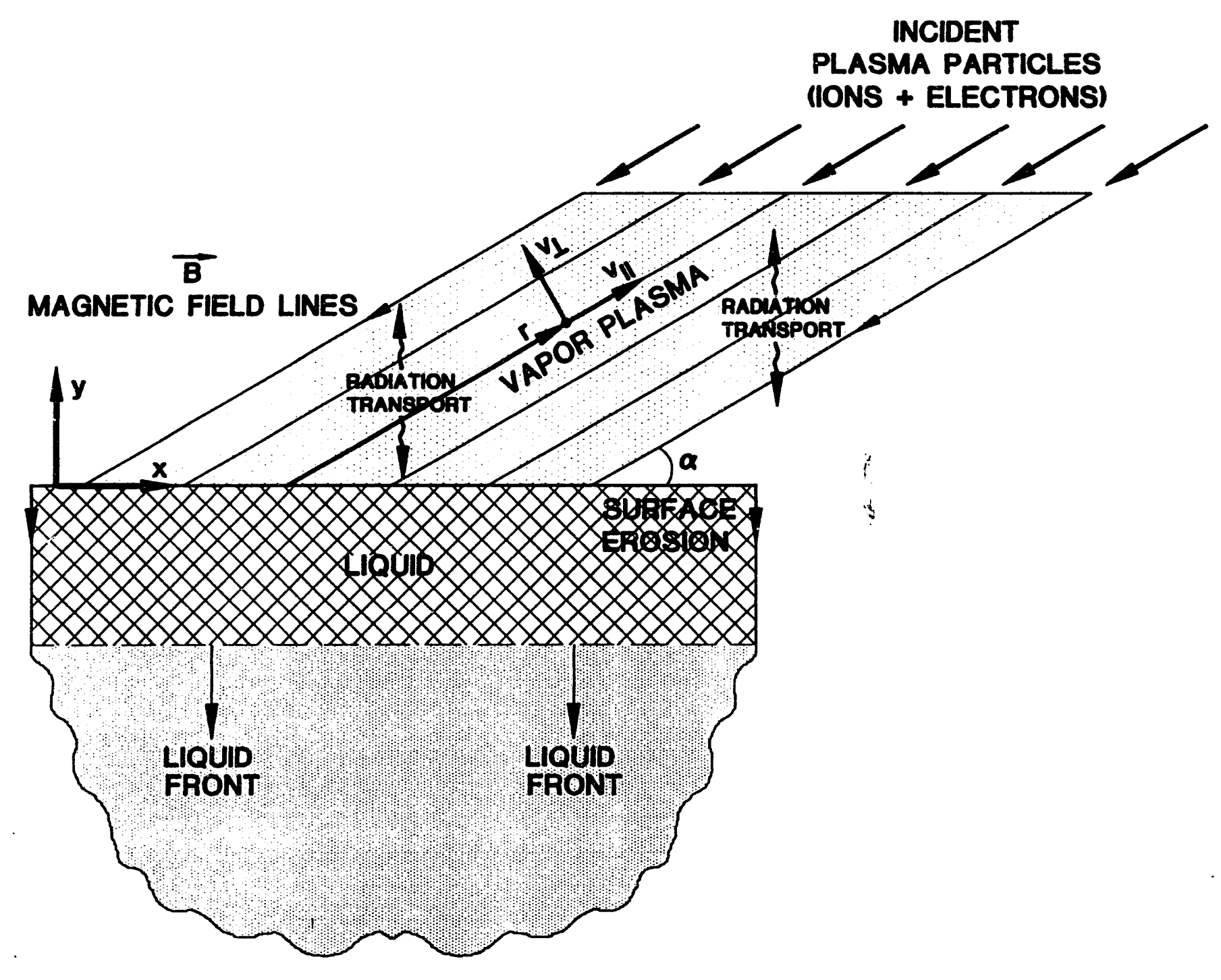

610047

$-i g$ (1) 


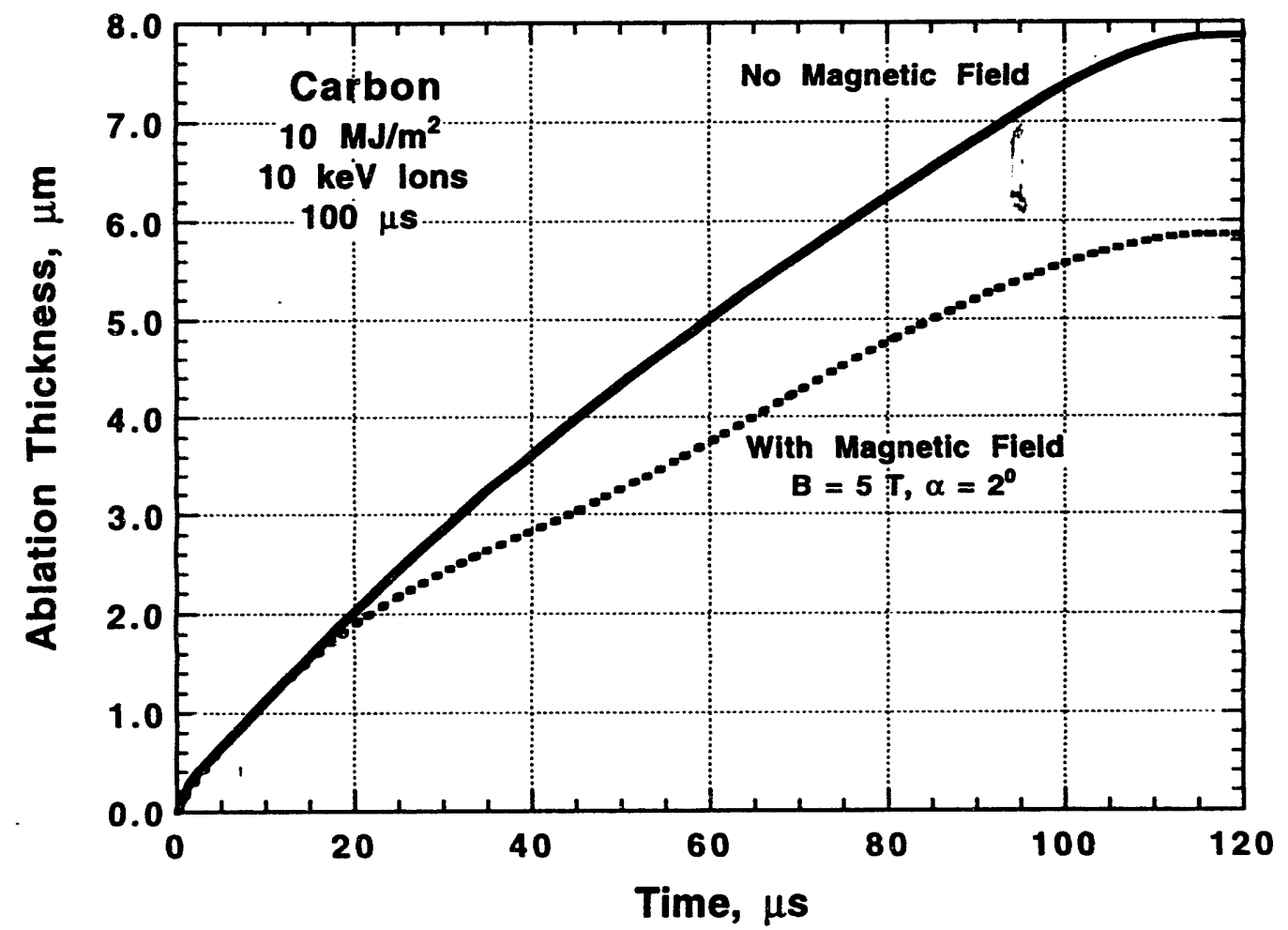

Fig(2) 


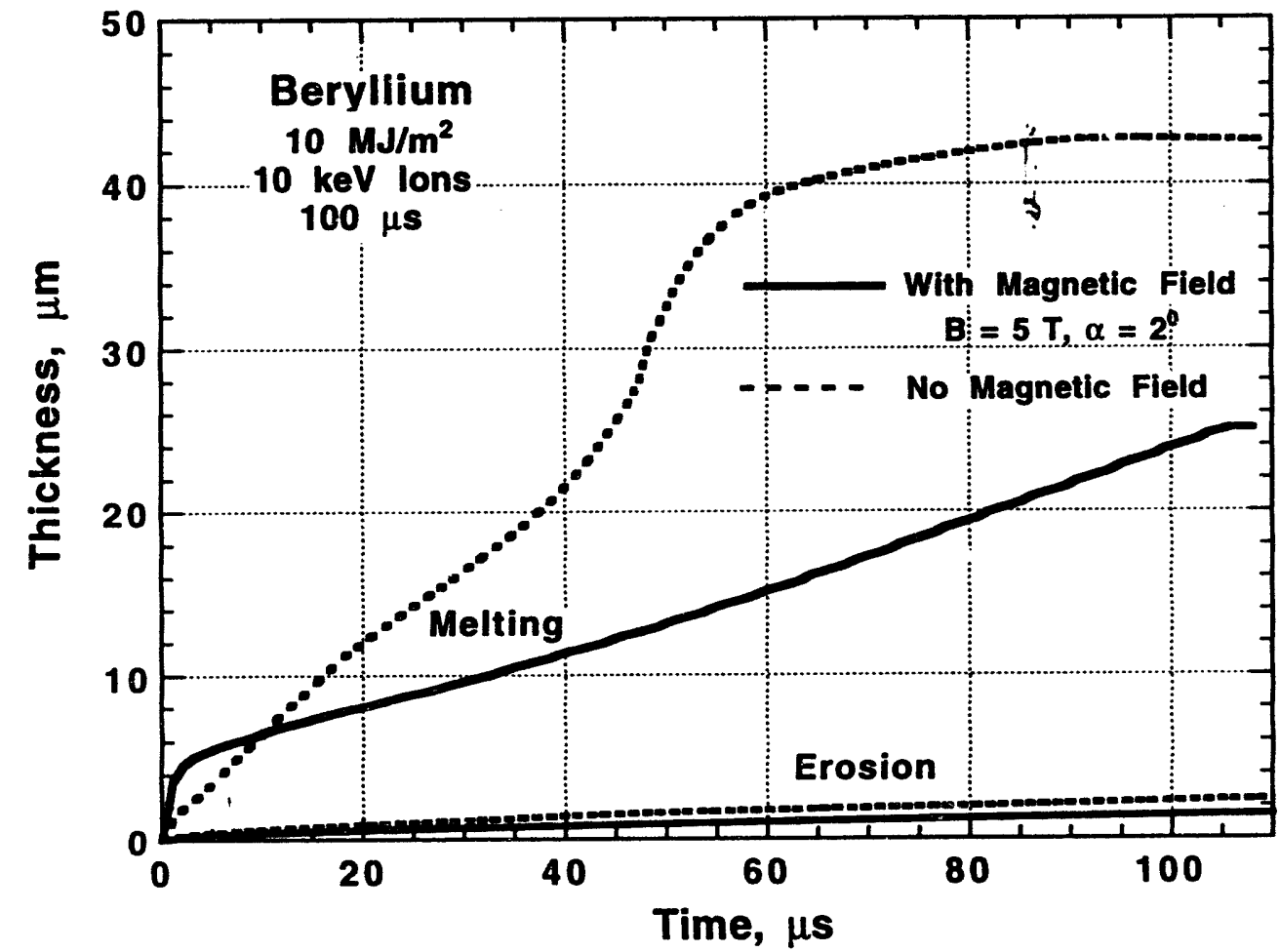

Fig (3) 


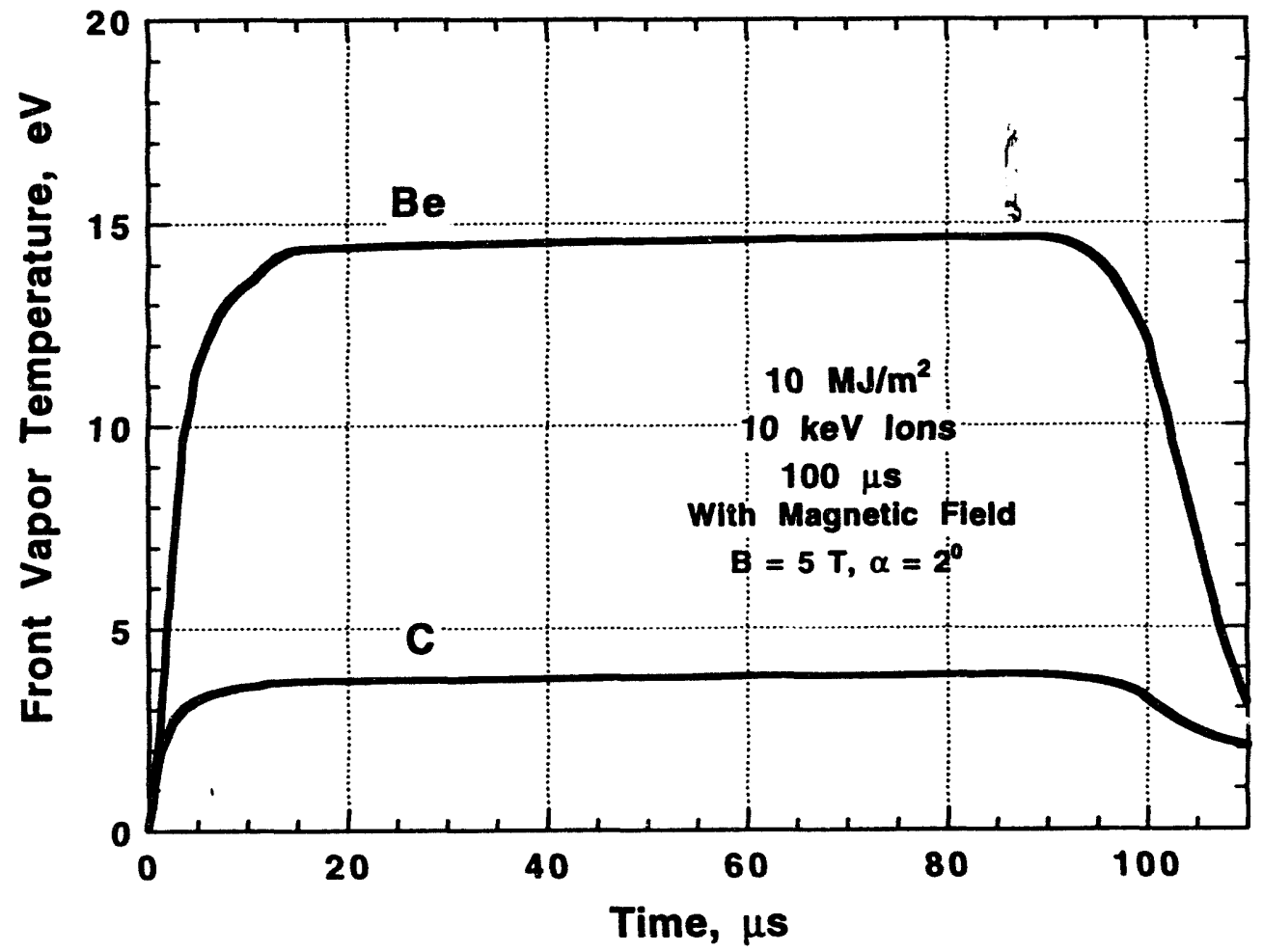

fig (4) 
(n)

4

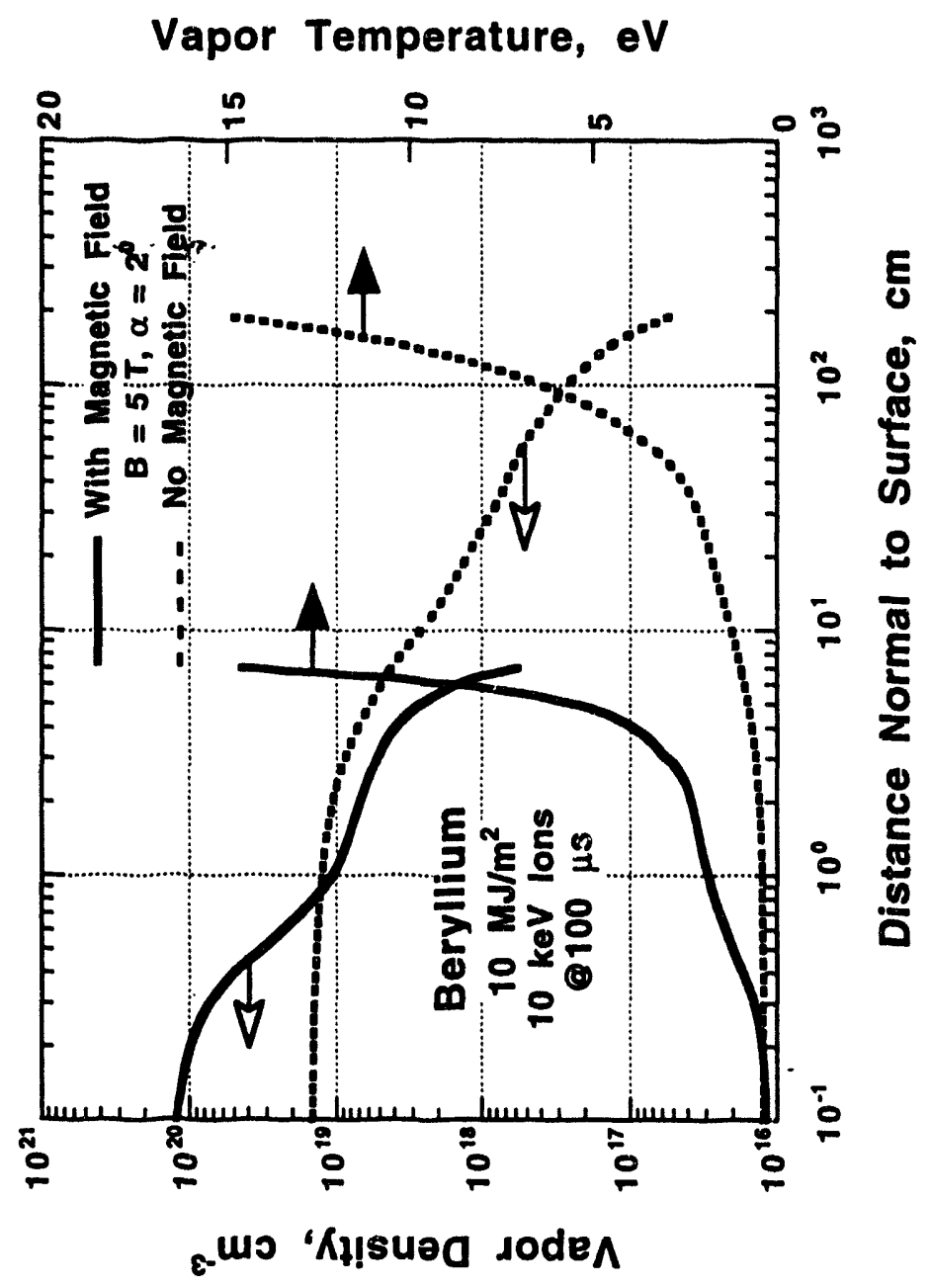




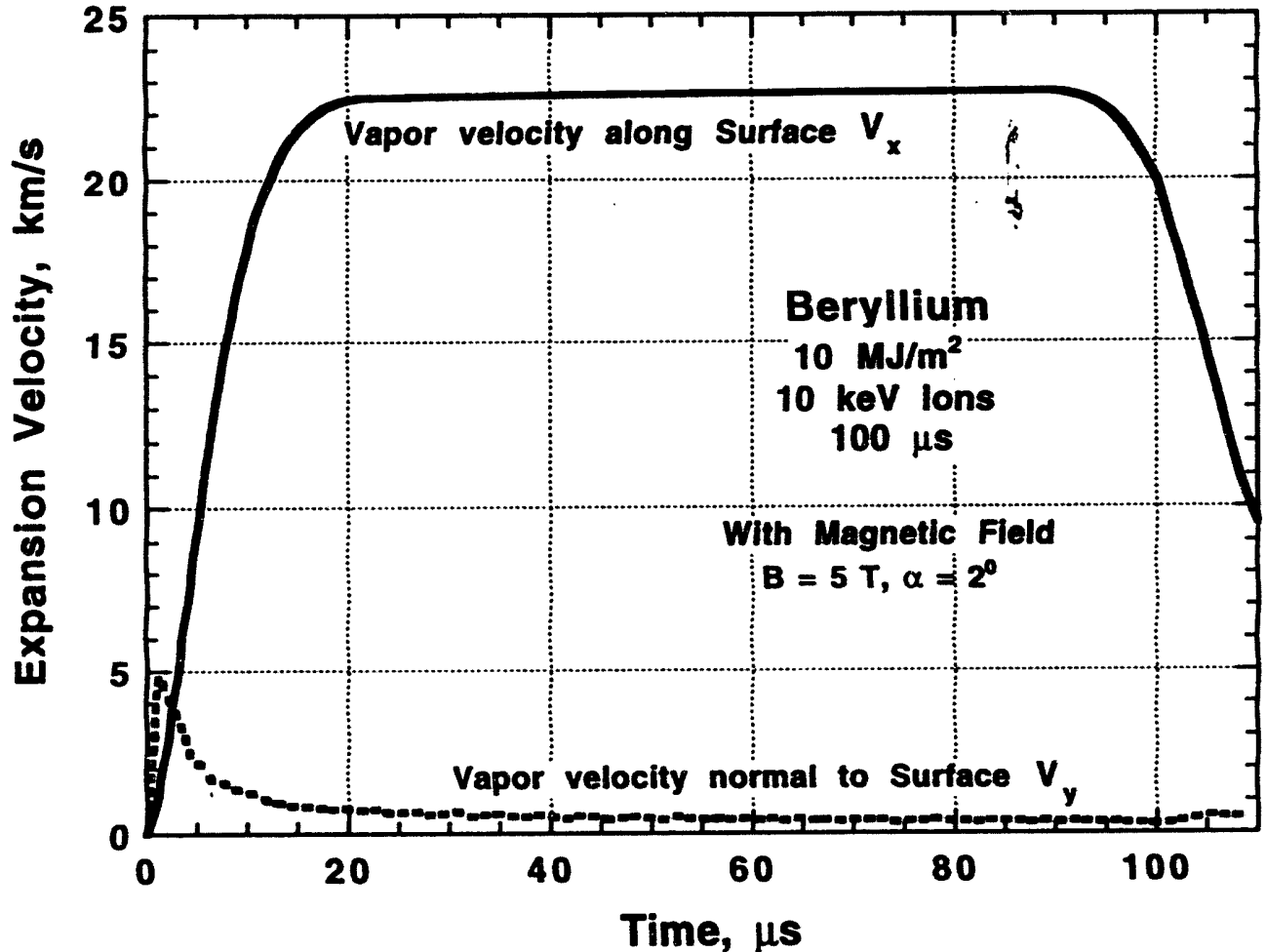

Fig (6) 


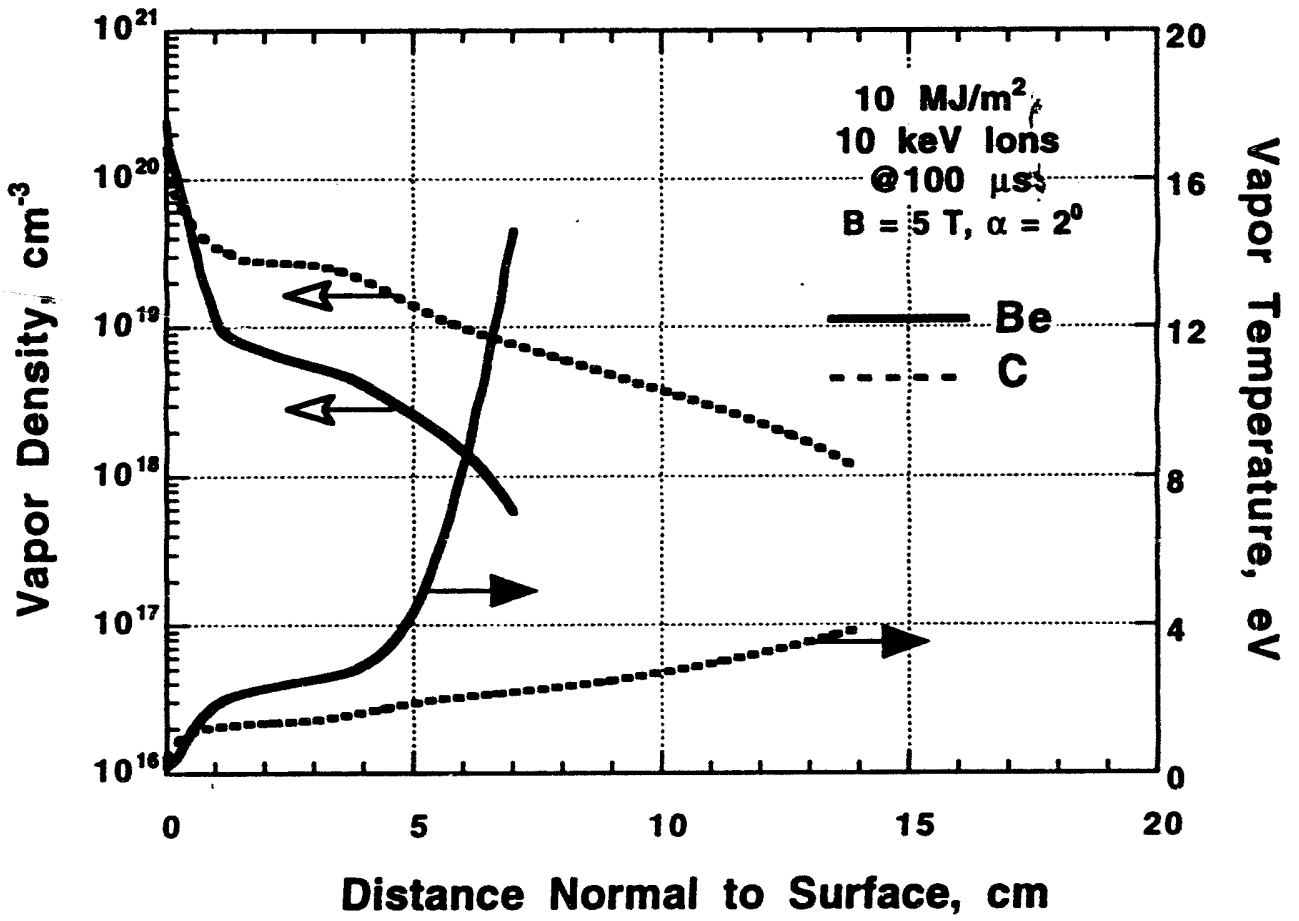

Fig 7 
$\infty$ 100

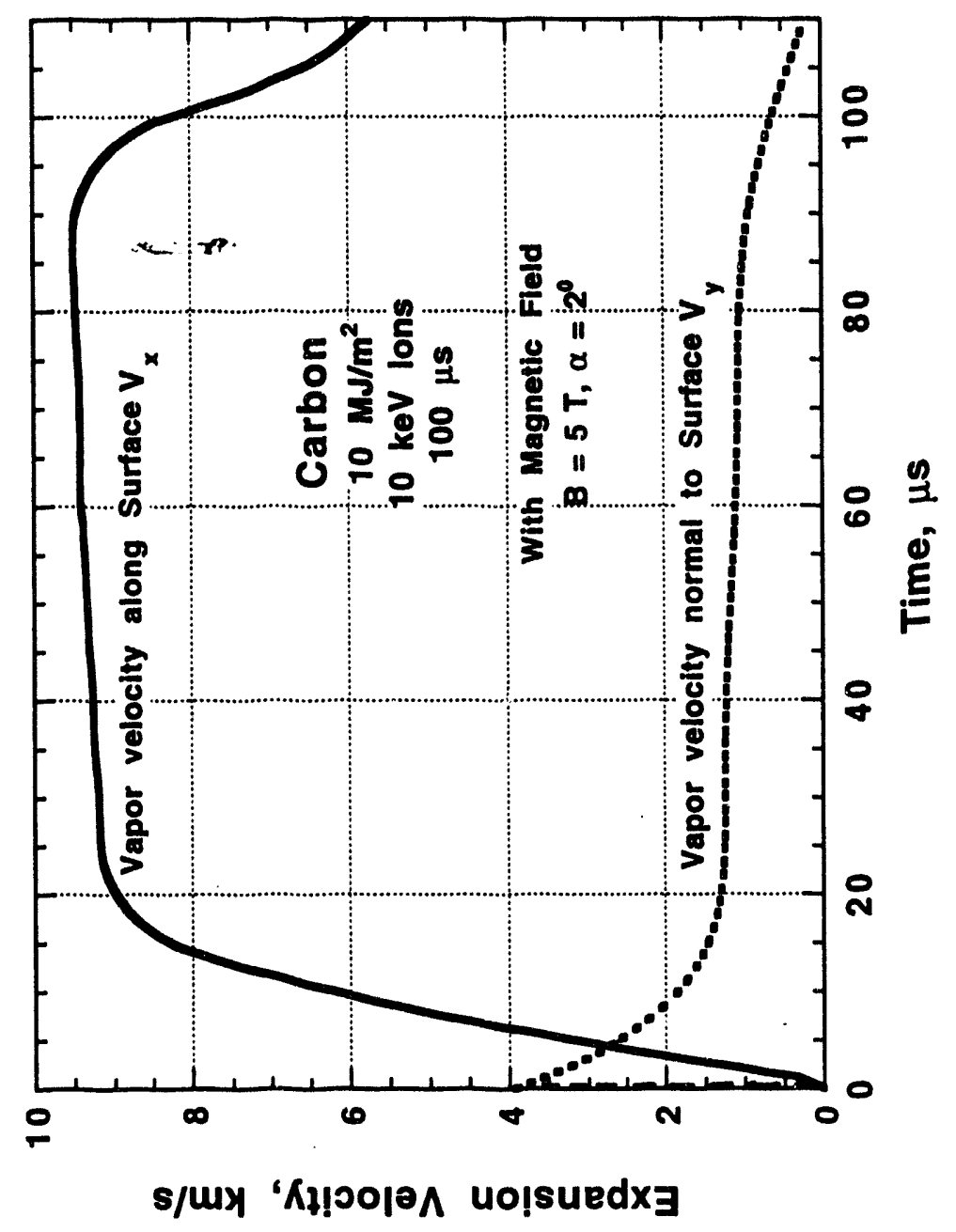




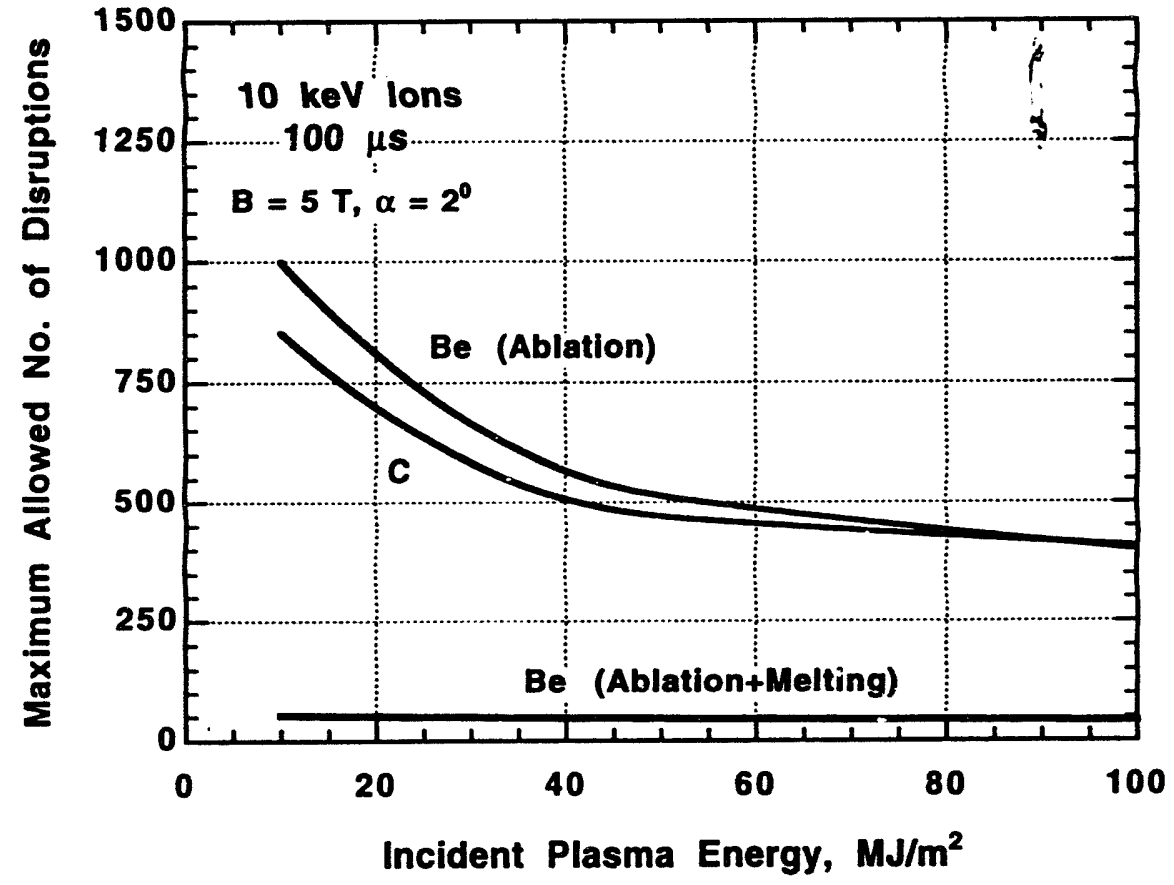

rig (9) 

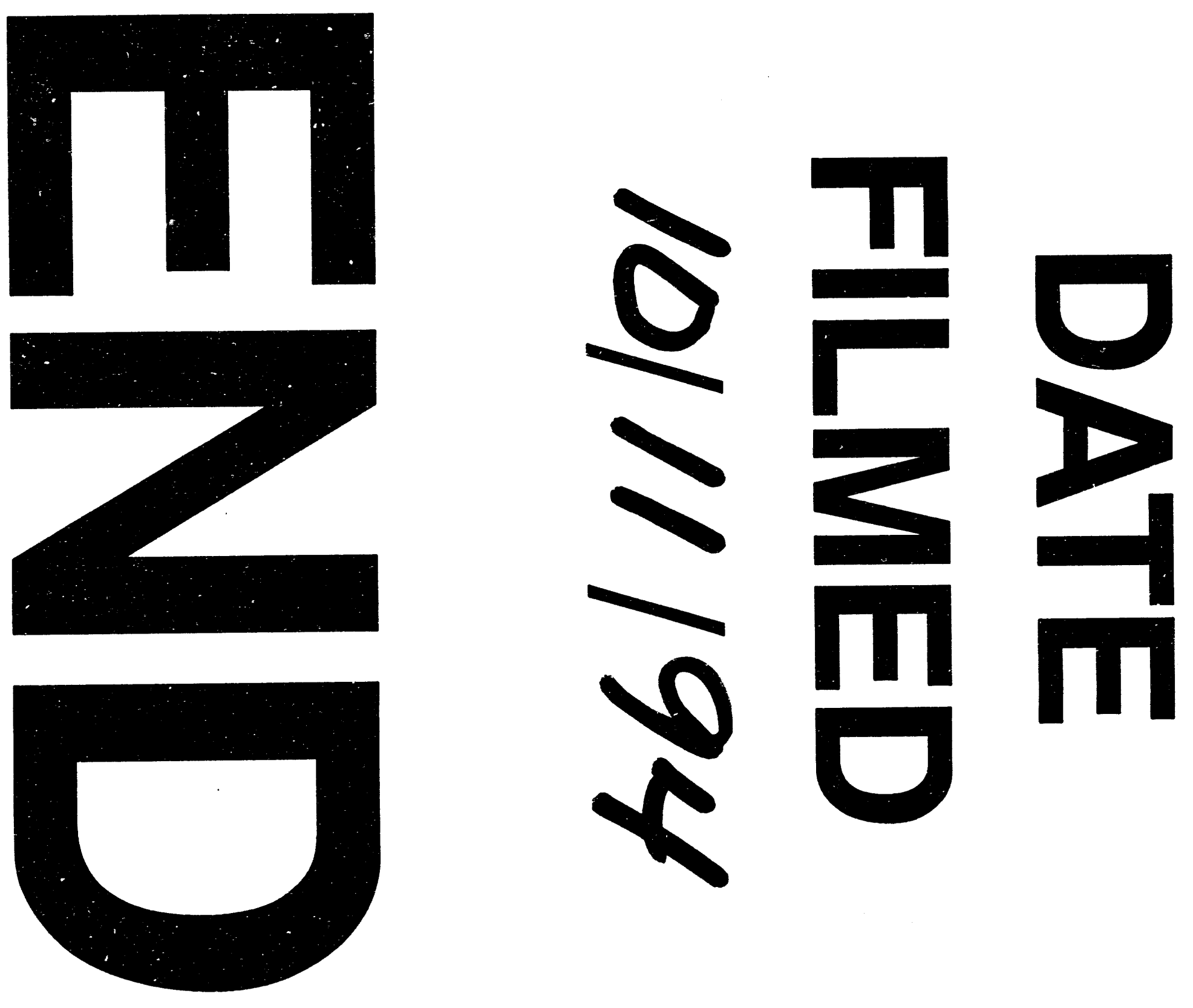
